\title{
Metastasis rates and sites after treatment for choroidal melanoma by proton beam irradiation or by enucleation
}

\author{
Chryssanthi Koutsandrea \\ Marilita M Moschos \\ Michael Dimissianos \\ Gerasimos Georgopoulos \\ loannnis Ladas \\ Michael Apostolopoulos \\ Department of Ophthalmology, \\ Athens University, Athens, Greece
}

\begin{abstract}
Purpose: To investigate tumor thickness and location, the interval of time between treatment for choroidal melanoma (proton beam irradiation or enucleation) and diagnosis of metastatic disease, and rates and sites of metastasis.

Design: Retrospective, interventional, noncomparative case series.

Methods: Follow-up of 152 patients with primary choroidal melanoma, between 1992 and 2006 (14 years). One hundred and twenty-one patients were treated with proton beam irradiation and 31 patients were treated with enucleation. Baseline and annual or semiannual ophthalmic examination, B-scan ultrasonography, systemic and laboratory evaluations (liver enzymes, chest X-ray, ultrasonography or magnetic resonance imaging of the liver) were performed according to a standard protocol.

Results: Nineteen patients (12.5\%) were diagnosed with metastasis during follow-up time after treatment for choroidal melanoma. Thirteen patients $(10.7 \%)$ of the irradiation group and 6 patients (19.3\%) of the enucleation group were diagnosed with metastasis. Eight patients (6.6\%) of the irradiation group and 5 patients $(16.1 \%)$ of the enucleation group were diagnosed with liver metastasis. Ocular complications after proton beam irradiation were recorded. Fifty-nine patients $(48.7 \%)$ of the irradiation group presented with cataract. Other complications were retinal detachment, retinopathy, vitreous haemorrhage, iris neovascularization, neovascular glaucoma, optic neuropathy, and corneal opacification.

Conclusions: In our series, $10.7 \%$ of the irradiation group and $19.3 \%$ of the enucleation group were diagnosed with metastasis. The liver was the most common site of metastasis in both groups. Cataract was the most common complication in the irradiation group.
\end{abstract}

Keywords: melanoma, metastasis, irradiation, enucleation

\section{Introduction}

Choroidal melanoma arises from melanocytes within the choroid. It is the most common primary intraocular cancer in adults. Because this cancer is often asymptomatic, it is often diagnosed after a routine eye examination, unless the patient's central vision is affected. Until about 1970, enucleation was the standard treatment. In the early 1970s, radiotherapy and other treatment approaches, with the goal of preserving the eye, became increasingly popular. External beam irradiation using charged particles, such as protons may conserve a useful level of vision, depending on the size of the tumor and its location with respect to the optic disc and macula. Protons deposit most of their energy near the end of their range, resulting in a uniform and highly localized dose being deposited largely in the tumor, sparing adjacent healthy tissue. Furthermore, as diagnostic aids and skills improved, misdiagnosis rates declined. Choroidal melanoma has a well-documented capacity to metastasize hematogenously. The liver is the most common site of metastatic disease (Einhorn et al 1974; Char 1978; Zakka et al 1980; Rajpal et al 1983; Diener-West et al 2001; 
Kath et al 1993; Bedikian et al 1995; COMS 2001, 2004, 2005). Diagnosis of metastasis is often followed by rapid physical decline of the patient and treatment is generally ineffective (Albert et al 1992). The median time from diagnosis of liver metastasis to death is approximately 6 months. After diagnosis of choroidal melanoma, patients are screened for metastasis 6-monthly or annually, using liver function tests (LFTs) and imaging studies. More recently routine screening by ultrasonography of the liver and a panel of LFTs have been recommended (Donoso et al 1985; Donoso et al 1985; Pach and Robertson 1986; COMS 1995; Hicks 1998; Eskelin et al 1999). Our primary aim was to study the time interval between a diagnosis of melanoma and a diagnosis of metastasis, and the rates and sites of metastasis during follow-up time in our patients. A secondary aim was to record ocular complications after proton beam irradiation (Seddon et al 1986).

\section{Methods}

One hundred and fifty-two eyes of 152 patients with primary choroidal melanoma, whose primary treatment was either proton beam irradiation or enucleation, were included in the study. The irradiation group included 121 patients. The enucleation group included 31 patients. Of these 152 patients, 69 were male and 83 female. The patients were treated between 1992 and 2006. Subjects with clinical evidence of metastatic disease at the time of treatment or prior to the treatment of their intraocular tumor were excluded. All patients gave written consent before treatment.

\section{Initial evaluation included Ophthalmic examination}

Slit-lamp examination, biomicroscopy, indirect ophthalmoscopy, fundus photographs and A-scan and B-scan ultrasonography. Tumor height was measured and used to classify a tumor as small ( $1 \mathrm{~mm}$ to $2.5 \mathrm{~mm}$ in apical height), medium ( $>2.5 \mathrm{~mm}$ to $10 \mathrm{~mm}$ ) or large ( $>10 \mathrm{~mm}$ in apical height). Tumor location and associated features, such as secondary retinal detachment and hemorrhage were recorded. The initial evaluation also included fluorescein and indocyanine green angiography, computed tomography (CT), and/or magnetic resonance imaging $(\mathrm{MRI})$.

\section{Systemic evaluation}

Complete blood count, liver function tests (aspartate aminotranspherase [AST], alanine aminotranspherase [ALT], alkaline phosphatase [APH], gamma-glutamyl transpeptidase $[\gamma-\mathrm{GT}]$, lactate dehydrogenase $[\mathrm{LDH}]$, carcinoembryonic antigen [CEA]). Ultrasound and CT or MRI evaluation of the abdominal organs (especially the liver) and chest radiograph.

Proton beam irradiation was performed at Paul Scherrer Institute (Villigen, Switzerland). The patients from the proton beam irradiation group were admitted approximately two weeks before the scheduled date of irradiation. In the surgical suite, the tumor was localized using transillumination and indirect ophthalmoscopy, and four tantalum rings measuring 2.5 millimeters in diameter were sutured to the sclera to serve as references marking the tumor edges. Tumors located too far anteriorly were diagrammed using precise measurements, without the tantalum rings.

During the irradiation sessions, the patient was seated and the head was immobilized using a custom-molded plastic mask. Lid retractors were used to remove the lid from the radiation field, and the patient was asked to fix his or her gaze on a light attached to the proton collimator. The eye was viewed on a closed circuit television camera using high magnification, to detect any deviation from the indicated direction of gaze. An imaging system was used to verify the eye's precise position during the treatment.

A computer planning program was used to target the proton beam. Provided with the necessary data, the computer generated a three-dimensional model of the eye and tumor, which could be viewed from any desired angle. At the time the model also calculated the best direction of gaze, ideal shape of the field defining apertures, proton range and range modulations, the structures lying in the path of the beam, and what the viewer should see in the alignment film used when aiming the beam. The radiation dose was 60 cobalt Gray equivalents delivered in 4 equal fractions.

Enucleation was performed at the Department of Ophthalmology of the University of Athens. The surgical technique consisted of peritomy for $360^{\circ}$ at the limbus. The rectus muscles were individually hooked and cut with scissors near their insertion into the sclera. The superior and inferior oblique muscles were hooked with two muscle hooks and cut. The enucleation scissors were placed between Tenon's capsule and the sclera, the optic nerve was cut and the eye removed. An orbital implant (hydroxyapatite sphere) was placed in the socket and the muscles were sutured to the implant.

Following enucleation, eyes were processed for histopathologic confirmation of the diagnosis at the Pathology Laboratory of Athens General Hospital. The patients were re-examined 6-monthly for the first 5 years and annually thereafter. Follow-up evaluation included: 


\section{Ophthalmic examination}

Slit-lamp examination, biomicroscopy, indirect ophthalmoscopy, fundus photographs and A-scan and B-scan ultrasonography. Tumor height was measured.

\section{Systemic evaluation}

Complete blood count, liver function tests (AST, ALT, APH, $\gamma$-GT, LDH, CEA). Ultrasound evaluation of the abdominal organs (especially the liver). In case of abnormal findings, CT or MRI scan of the liver and/or biopsy or cytologic tests were required to confirm or rule out metastatic disease.

Ocular (anterior and posterior segment) complications of proton beam irradiation were recorded.

\section{Results}

Tumor thickness according to B-scan ultrasonography, tumor location according to binocular indirect ophthalmoscopy, and Goldmann triple mirror examination were recorded. In the enucleation group the affected eye was the right one in $18(58 \%)$ patients and the left one in 13 (42\%). Tumor thickness ranged from $4.6 \mathrm{~mm}$ to $13.4 \mathrm{~mm}$ with a mean value of $9.7 \mathrm{~mm}$. Tumor location was superior in $6(19.4 \%)$ patients, inferior in $4(12.9 \%)$ patients, temporal in $7(22.6 \%)$ patients, nasal in $4(12.9 \%)$ patients, superior/temporal in $2(6.4 \%)$ patients, inferior/temporal in $4(12.9 \%)$ patients, and inferior/nasal in $4(12.9 \%)$ patients (Figure 1).

In the irradiation group, the affected eye was the right one in $57(47 \%)$ patients and the left one in $64(53 \%)$. Tumor thickness ranged from $2.6 \mathrm{~mm}$ to $13.4 \mathrm{~mm}$ with a mean value of $7.3 \mathrm{~mm}$. Tumor location was superior in $22(18.2 \%)$ patients, inferior in $18(14.9 \%)$ patients, temporal in $20(16.5 \%)$ patients, nasal in $8(6.6 \%)$ patients, superior/temporal in $13(10.7 \%)$ patients, superior/nasal in $4(3.3 \%)$ patients, inferior/temporal in $26(21.5 \%)$ patients and inferior/nasal in $10(8.3 \%)$ patients (Figure 1).

Of the 152 patients in our study, 31 were treated by enucleation between 1992 and 2006 (16 males, 15 females). The average age of patients was 60.8 years. The median follow-up period was 60 months ( 5 years). Of the 31 patients, $6(19.3 \%)$ were diagnosed with metastasis ( 5 males, 1 female). The metastatic sites included: liver ( 5 patients, $16.1 \%$ of enucleation group, $83.3 \%$ of the patients with metastasis) and skin (1 patient, $3.2 \%$ of enucleation group, $16.7 \%$ of the patients with metastasis) (Figure 2).

The median interval time from diagnosis of choroidal melanoma to diagnosis of metastasis was 29.5 months
(2.46 years). At 5 years, 5 patients were diagnosed with metastasis (16.1\% of enucleation group, $83.3 \%$ of patients with metastasis).

Between 1992 and 2006, 121 patients were treated by proton beam irradiation ( 53 males, 68 females). The average age of patients was 64.2 years. The median follow-up period was 79.6 months (6.6 years). Thirteen patients $(10.7 \%)$ were diagnosed with metastasis ( 7 males, 6 females). The metastatic sites included: liver ( 8 patients, $6.6 \%$ of irradiation group, $61.5 \%$ of the patients with metastasis), lung ( 2 patients, $1.6 \%$ of irradiation group, $15.4 \%$ of the patients with metastasis), brain (1 patient, $0.8 \%$ of irradiation group, $7.7 \%$ of the patients with metastasis), thyroid (1 patient, $0.8 \%$ of irradiation group, $7.7 \%$ of the patients with metastasis), and colon ( 1 patient, $0.8 \%$ of irradiation group, $7.7 \%$ of the patients with metastasis) (Figure 2). The median interval of time from diagnosis of choroidal melanoma to the diagnosis of metastasis was 44.8 months (3.7 years). At 5 years, 10 patients were diagnosed with metastasis $(8.3 \%$ of irradiation group, $76.9 \%$ of patients with metastasis).

Ocular complications were recorded after proton beam irradiation, according to slit-lamp examination, biomicroscopy, indirect ophthalmoscopy, fundus photographs, and A-scan and B-scan ultrasonography. Anterior segment complications were recorded in 99 (81.8\%) patients and posterior segment complications in $71(58.7 \%)$ patients. Cataract was recorded in $59(48.7 \%)$ patients, iris neovascularization in 17 (14\%) patients, neovascular glaucoma in $14(11.6 \%)$ patients, and corneal opacification in $9(7.4 \%)$ patients. Secondary retinal detachment was recorded in 25 (20.7\%) patients, retinopathy in 17 (14\%) patients, vitreous hemorrhage in 17 (14\%) patients and optic neuropathy in $12(9.9 \%)$ patients (Figure 3).

\section{Discussion}

For more than a century, enucleation has been the standard treatment for choroidal melanoma. However, concerns about enucleation, especially possible tumor spread caused by manipulation of the eye during surgery, plus vision loss and the poor cosmetic outcome, have made irradiation therapy a popular alternative. When patients are deciding on their treatment for choroidal melanoma, the major influences on their decision are fear of death due to cancer, and concerns about the prognosis of vision in the affected eye. Many ophthalmologists assume that better results can be achieved with irradiation therapy, because this form of treatment potentially allows the retention of some vision and avoids the perception of disfigurement. 


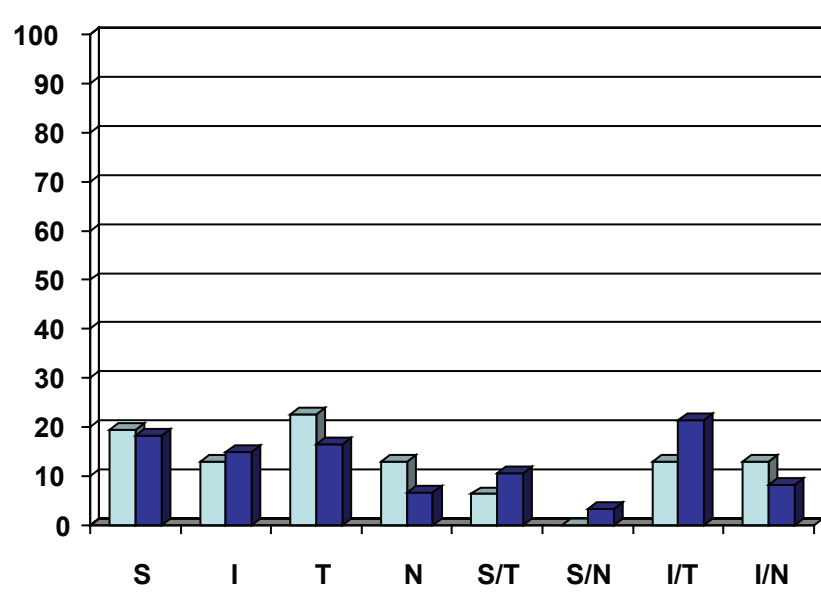

\begin{tabular}{|l|}
$\square$ Enucleation \\
$\square$ Irradiation \\
\hline
\end{tabular}

Figure I Chart demonstrating tumor location of the enucleation group (light blue) and the irradiation group (dark blue).

Abbreviations: S, superior quadrant; I, inferior quadrant; T, temporal quadrant; $\mathrm{N}$, nasal quadrant; $\mathrm{S} / \mathrm{T}$, superior/temporal quadrant; I/T, inferior/temporal quadrant; I/N, inferior/nasal quadrant.

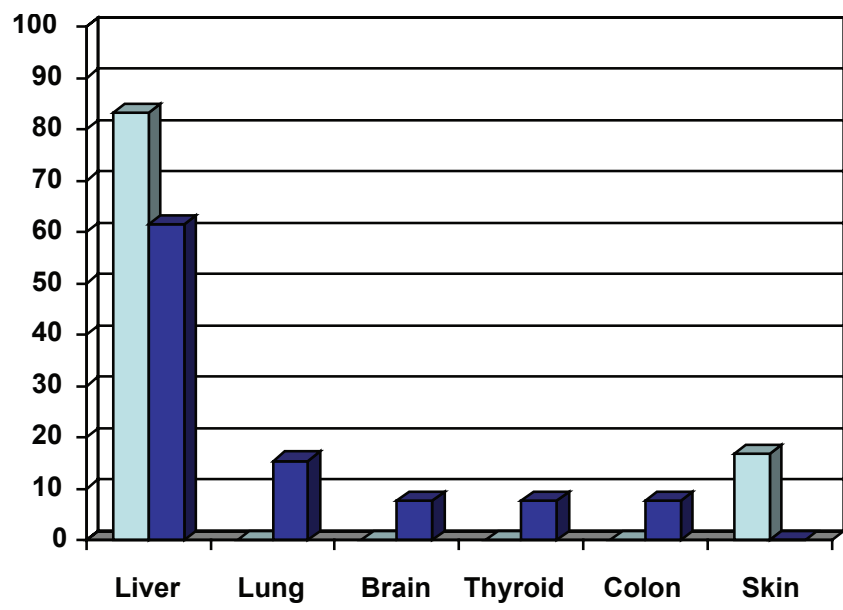

$\square$ Enucleation

Irradiation

Figure 2 Metastatic sites of the two treatment groups. In the enucleation group the metastatic sites appear in light blue, and in the irradiation group appear in dark blue.

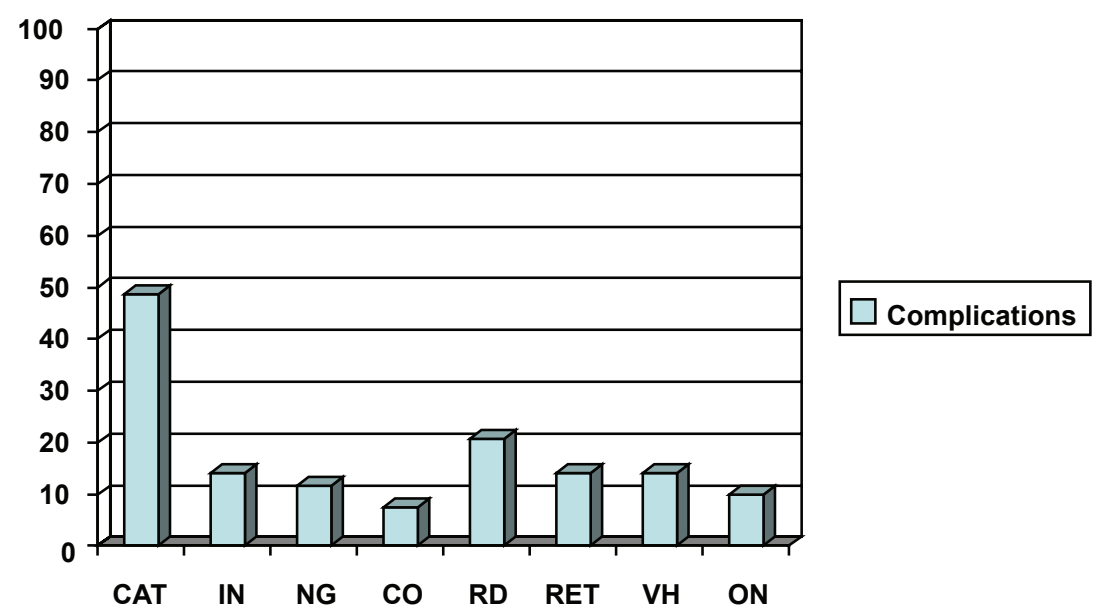

Figure 3 The chart clearly demonstrates the complications of proton beam irradiation.

Abbreviations: CAT, cataract; IN, iris neovascularization; NG, neovascular glaucoma; CO, corneal opacification; RD, retinal detachment; RET, retinopathy; VH, vitreous hemorrhage; ON, optic neuropathy. 
A 1986 study (Gragoudas et al 1987) showed a $20 \%$ cumulative risk of developing metastasis 5 years after irradiation, based on 128 patients followed for a median of 5.4 years. In a study (Gragoudas et al 1988) of 780 eyes (which included many of the 128 noted above) followed for a median of 2.2 years, $8 \%$ of that study population actually developed metastases, the median time to detection being 2.1 years. Based on Kaplan-Meier probability curves, the same 5-year estimate of a $20 \%$ cumulative risk of metastasis was determined. Eighty percent to $90 \%$ of metastases from uveal melanoma occurred in the liver in those patients, less common sites being the skin and lung.

In our study we recorded the tumor location, the rates and sites of metastasis, and the time from diagnosis of melanoma to diagnosis of metastasis. In the enucleation group, the most common location was temporal (22.6\%; 7 patients). Six patients (19.3\%) were diagnosed with metastasis. Liver was the most common site $(83.3 \%)$. The median time from diagnosis of choroidal melanoma to diagnosis of metastasis was 29.5 months (2.46 years). Five of the 6 patients with metastasis $(83.3 \%)$ were diagnosed within the first 5 years after treatment (enucleation). In the irradiation group, the most common location was inferior/temporal $(21.5 \%$; 26 patients). Thirteen patients $(10.7 \%)$ were diagnosed with metastasis. The liver was the most common site $(61.5 \%)$. The median time from diagnosis of choroidal melanoma to diagnosis of metastasis was 44.8 months (3.7 years). Ten of the 13 patients with metastasis $(76.9 \%)$ were diagnosed within the first 5 years after treatment (irradiation).

Important prognostic factors for death from metastatic melanoma include the size of the tumor (the larger the tumor, the worse the prognosis), the location of the tumor (tumors within the ciliary body are associated with a poorer prognosis than those confined to the choroid), the age of the patient at the time of diagnosis (the older the patient, the worse the short-term survival prognosis) and extrascleral tumor extension (Augsburger and Gamel 1990). Patients with choroidal melanoma are advised to undergo periodic examination, blood tests for liver enzyme levels and imaging studies, such as chest radiographs and ultrasonography or MRI of the liver. We agree with other studies (Hicks 1998; Eskelin et al 1999) that periodic screening for metastatic uveal melanoma is primarily beneficial, when effective treatment is available or when patients are eligible as candidates for clinical trials of promising treatment. Such therapies are needed given the potentially frequent occurrence of micrometastasis at the time of diagnosis of choroidal melanoma and the high incidence of metastasis detected clinically after diagnosis of the primary tumor.

Many treated eyes eventually lose a substantial amount of vision, as a consequence of complications after radiation therapy. Factors that influence how much vision will be lost include the location of the posterior edge of the tumor relative to the optic disc and fovea, the visual acuity before treatment, the presence or absence of macular retinal detachment, and the thickness of the tumor. Some systemic factors, such as diabetes mellitus, may also worsen a patient's prognosis for retention of useful vision (Seddon et al 1986). Retinal detachment, optic neuropathy, radiation retinopathy, neovascular glaucoma (Kim et al 1986; Summanen et al 1996; Daftari et al 1997), vitreous hemorrhage, and cataract (Meecham et al 1994; Gragoudas et al 1995) have emerged consistently as causes of visual loss in proton beam-irradiated eyes. Establishing which of these conditions is most influential in any given case may cause difficulty, as they may occur in the same patient, in whom the presence of a cataract may further hamper a view of the fundus. In our study, irradiation-related complications involving the anterior segment, which contributed to a decline in visual acuity or eye loss, included cataract (59 patients; $48.7 \%$ ), neovascular glaucoma (14 patients; $11.6 \%$ ), and corneal neovascularization and opacification ( 9 patients; $7.4 \%$ ).

Gragoudas and colleagues (1995) showed that $42 \%$ of 383 patients who received proton beam irradiation for uveal melanoma, developed new posterior subcapsular opacities in direct proportion to the dose of radiation received by the lens in the irradiated eye. This result was determined by a computer-generated dose-volume assessment routinely calculated at the time of irradiation. Cortical and sclerotic opacities were found to be correlated only with age in this study. Cataract extraction is feasible in these patients and can restore vision in some (Augsburger and Shields 1985; Fish et al 1991; Gragoudas et al 1992). Panretinal photocoagulation can substantially reduce the severity of neovascular glaucoma and reduce the risk of enucleation by half (Kim et al 1986).

In our study, posterior segment complications included secondary retinal detachment (25 patients; $20.7 \%$ ), radiation retinopathy (17 patients; $14 \%$ ), vitreous hemorrhage (17 patients; 14\%), and radiation neuropathy (12 patients; 9.9\%). Maculopathy affects central vision (Gragoudas et al 1992; Gunduz et al 1999; Guyer et al 1992), whereas papillopathy can lead to total loss of vision (Guyer et al 1992; Gragoudas et al 1999). Some eyes eventually become severely painful, usually because of neovascular glaucoma. Patients who develop a blind, painful eye must undergo enucleation (Kellner et al 1993). 
Preservation of the eye and useful vision in many patients are the obvious advantages of conservative treatment with radiation compared with enucleation. However questions have been raised regarding the efficacy of this treatment in limiting metastatic spread of the tumor. Observational studies (Egan et al 1989; Seddon et al 1990; Augsburger et al 1986) comparing irradiation with enucleation have indicated no significant differences in survival rates. In the Collaborative Ocular Melanoma Study (Adams et al 1998), overall 5-year mortality rates were comparable in patients treated by enucleation and by plaque radiotherapy (iodine 125). Proton irradiation is highly successful in achieving local control of intraocular melanoma. Many patients maintain some degree of function in the eye for long periods after treatment. Overall rates of metastatic disease are comparable to those observed after enucleation; thus, enucleation should be limited to patients with large tumors in whom the eye is unlikely to be salvaged by irradiation. Doubtless continued refinement of current methods, and development of new techniques, will further improve the outlook of patients with uveal melanomas.

\section{Conclusion}

In our series, $10.7 \%$ of the irradiation group and $19.3 \%$ of the enucleation group were diagnosed with metastasis. This difference is attributed to the larger tumor dimensions in patients of the enucleation group, given the observed correlation between increased metastasis rate and increased size of choroidal melanoma. The liver was the most common site of metastasis in both groups. Prognosis after metastatic disease remains poor. Effective methods are needed to prevent, diagnose, and treat metastasis from choroidal melanoma.

\section{Disclosure}

The authors report no conflicts of interest in this work.

\section{References}

Adams K, Abramson D, Ellsworth R, et al. 1988. Cobalt plaque versus enucleation for uveal melanoma: comparison of survival rates. $\mathrm{Br} J$ Ophthalmol, 72:494-7.

Albert DM, Niffennegger AS, Willson JKV, et al. 1992. Treatment of metastatic uveal melanoma: review and recommendations. Surv Ophthalmol, 36:429-38.

Augsburger J, Gamel J, Sardi V, et al. 1986. Enucleation vs cobalt plaque radiotherapy for malignant melanomas of the choroid and ciliary body. Arch Ophthalmol, 104:655-61.

Augsburger JJ and Gamel JW. 1990. Clinical prognostic factors in patients with posterior uveal malignant melanoma. Cancer, 66:1596-600.

Augsburger JJ and Shields JA. 1985. Cataract surgery following cobalt60 plaque radiotherapy for posterior uveal malignant melanoma. Ophthalmology, 92:815-22.
Bedikian AY, Legha SS, Mavligit G, et al. 1995. Treatment of uveal melanoma metastatic to the liver. Cancer, 76:1665-70.

Char DH. 1978. Metastatic choroidal melanoma. Am J Ophthalmol, 86:76-80.

[COMS] Collaborative Ocular Melanoma Study Group. 1995. COMS manual of procedures, Accession No. PB95-179693.

[COMS] Collaborative Ocular Melanoma Study Group. 2001. Assessment of metastatic disease status at death in 435 patients with large choroidal melanoma in the COMS. COMS Report No.15. Arch Ophthalmol, 119:670-6.

[COMS] Collaborative Ocular Melanoma Study Group. 2004. Screening for metastasis from choroidal melanoma: COMS Report No. 23. J Clin Oncol, 22:2438-44.

[COMS] Collaborative Ocular Melanoma Study Group. 2005. Development of metastatic disease after enrollment in the COMS trials for treatment of choroidal melanoma. COMS Report No. 26. Arch Ophthalmol, 123:1639-43.

Daftari IK, Char DH, Verhey LJ, et al. 1997. Anterior segment sparing to reduce charged particle radiotherapy complications in uveal melanoma. Int J Radiat Oncol Biol Phys, 39:997-1010.

Diener-West M, Earle JD, Fine SL, et al. 2001. The COMS randomized trial of iodine 125 brachytherapy for choroidal melanoma, III: initial mortality findings. COMS Report No. 18. Arch Ophthalmol, 119:969-82.

Donoso LA, Berd D, Augsburger JJ, et al. 1985. Metastatic uveal melanoma: Pretherapy serum liver enzyme and liver scan abnormalities. Arch Ophthalmol, 103:796-8.

Donoso LA, Shields JA, Augsburger JJ, et al. 1985. Metastatic uveal melanoma: Diffuse hepatic metastasis in a patient with concurrent normal serum liver enzyme levels and liver scan. Arch Ophthalmol, 103:758.

Egan KM, Gragoudas ES, Seddon JM, et al. 1989. The risk of enucleation after proton beam irradiation of uveal melanoma. Ophthalmology, 96:1377-83.

Einhorn LH, Burgess MA, Gottlieb JA, et al. 1974. Metastatic patterns of choroidal melanoma. Cancer, 34:1001-4.

Eskelin S, Pyrhonen S, Summanen P, et al. 1999. Screening for metastatic malignant melanoma of the uvea revisited. Cancer, 85:1151-9.

Fish GE, Jost BF, Snyder WI, et al. 1991. Cataract extraction after brachytherapy for malignant melanoma of the choroid. Ophthalmology, 98:619-22.

Gragoudas ES, Seddon JM, Egan KM, et al. 1987. Long-term results of proton beam irradiated uveal melanomas. Ophthalmology, 94:349-53.

Gragoudas ES, Seddon JM, Egan KM, et al. 1988. Metastasis from uveal melanoma after proton beam irradiation. Ophthalmology, 95:992-9.

Gragoudas ES, Egan KM, Arrigg PG, et al. 1992. Cataract extraction after proton beam irradiation for malignant melanoma of the eye. Arch Ophthalmol, 110:475-79.

Gragoudas ES, Egan KM, Walsh SM, et al. 1995. Lens changes after proton beam irradiation for uveal melanoma. Am J Ophthalmol, 119:157-64.

Gragoudas ES, Li W, Lane AM, et al. 1999. Risk factors for radiation maculopathy and papillopathy after intraocular irradiation. Ophthalmology, 106:1571-8.

Guyer D, Mukai S, Egan K, et al. 1992. Radiation maculopathy after proton beam irradiation for choroidal melanoma. Ophthalmology, 99:1278-85.

Gunduz K, Shields CL, Shields JA, et al. 1999. Radiation retinopathy following plaque radiotherapy for posterior uveal melanoma. Arch Ophthalmol, 117:609-14.

Hicks C, Foss AJE, Hungerford JL, et al. 1998. Predictive power of screening tests for metastasis in uveal melanoma. Eye, 12:945-8.

Kath R, Hayungs J, Bornfeld N, et al. 1993. Prognosis and treatment of disseminated uveal melanoma. Cancer, 72:2219-23.

Kellner U, Bornfeld N, Foerster MH, et al. 1993. Radiation-induced optic neuropathy following brachytherapy of uveal melanomas. Graefes Arch Clin Exp Ophthalmol, 231:267-70. 
Kim M, Char D, Castro J, et al. 1986. Neovascular glaucoma after helium ion irradiation for uveal melanoma. Ophthalmology, 93:189-93.

Meecham W, Char D, Kroll S, et al. 1994. Anterior segment complications after helium ion radiation therapy for uveal melanoma: radiation cataract. Arch Ophthalmol, 112:197-203.

Pach JM and Robertson DM. 1986. Metastasis from untreated uveal melanoma. Arch Ophthalmol, 104:1624-5.

Rajpal S, Moore R, Karakousis CP, et al. 1983. Survival in metastatic ocular melanoma. Cancer, 52:334-6.

Seddon JM, Gragoudas ES, Egan KM, et al. 1990. Relative survival rates after alternative therapies for uveal melanoma. Ophthalmology, 97:769-77.
Seddon JM, Gragoudas ES, Polivogianis L, et al. 1986. Visual outcome after proton beam irradiation of uveal melanoma. Ophthalmology, 93:666-74.

Summanen P, Immonen I, Kivela T, et al. 1996. Radiation related complications after ruthenium plaque radiotherapy of uveal melanoma. Br J Ophthalmol, 80:732-9.

Zakka KA, Foos RY, Omphroy CA, et al. 1980. Malignat melanoma: Analysis of an autopsy population. Ophthalmology, 87:549-56. 
